\title{
Les « Ententes sur les répercussions et les avantages » et le bien-être des communautés \\ Des occasions ratées?
}

\section{Impact and Benefit Agreements and community well-being : A missed opportunity? \\ "Acuerdos sobre las ventajas y repercusiones " el bienestar de las comunidades: ¿Ocasiones perdidas?}

\author{
Cathleen Knotsch, Peter Siebenmorgen et Ben Bradshaw
}

Volume 40, numéro 3, 2010

URI : https://id.erudit.org/iderudit/1009369ar

DOI : https://doi.org/10.7202/1009369ar

Aller au sommaire du numéro

Éditeur(s)

Recherches amérindiennes au Québec

ISSN

0318-4137 (imprimé)

1923-5151 (numérique)

Découvrir la revue

Citer cet article

Knotsch, C., Siebenmorgen, P. \& Bradshaw, B. (2010). Les « Ententes sur les répercussions et les avantages » et le bien-être des communautés : des occasions ratées ? Recherches amérindiennes au Québec, 40(3), 59-68. https://doi.org/10.7202/1009369ar

\section{Résumé de l'article}

Depuis deux décennies, de plus en plus d'ententes sur les répercussions et les avantages (ERA) ont été négociées entre les minières et les communautés autochtones ou leurs représentants régionaux. Ces ententes sont perçues comme un outil très utile pour promouvoir le développement économique et améliorer certaines conditions socioéconomiques des communautés, plus particulièrement l'accroissement du revenu. Il n'est pas toujours évident toutefois que les communautés ainsi enrichies soient choyées au point de vue santé et bien-être communautaire. Plus frustrant encore, il n'y a qu'une minorité d'ERA qui se préoccupent directement de santé et de bien-être communautaire car la plupart sont conçues plutôt pour apporter des avantages en présumant que ceux-ci amélioreront le bien-être en général. C'est trompeur et insuffisant. En prenant pour exemple les ERA signées par les organisations inuites du Canada, le présent article reflète celui de Knotsch et Warda (2009) dans lequel les auteurs proposent plus de recherches sur les ERA et sur le bien-être des communautés. De plus, nous suggérons ici que les négociateurs des ERA mettent l'accent sur les questions et les besoins reliés à la santé communautaire en les intégrant dans les futures ententes.
Tous droits réservés @ Recherches amérindiennes au Québec, 2010

Ce document est protégé par la loi sur le droit d'auteur. L'utilisation des services d'Érudit (y compris la reproduction) est assujettie à sa politique d'utilisation que vous pouvez consulter en ligne.

https://apropos.erudit.org/fr/usagers/politique-dutilisation/ 


\section{Les « Ententes sur les répercussions et}

les avantages » et le bien-être des communautés Des occasions ratées?

\section{Cathleen Knotsch, \\ Peter}

Siebenmorgen

et

\section{Bradshaw*}

Traduit de

I'anglais par

Paule Lamarche

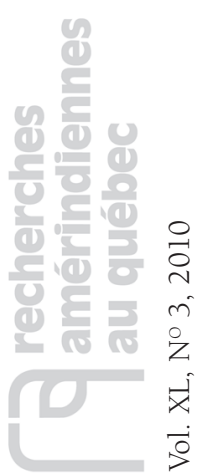

TLEST RECONNU DEPUIS LONGTEMPS que les Inuits, les nations amérindiennes et les Métis sont moins en santé que le reste de la population canadienne. De façon générale, les peuples autochtones sont plus vulnérables, et les déterminants sociaux, occupationnels et environnementaux sont reliés de façon particulière aux questions de santé (Adelson 2005; Montenegro et Stephens 2006; Richmond et Ross 2009). Cela est particulièrement vrai pour les Inuits vivant dans le territoire Inuit Nunangat $^{1}$ - où l'espérance de vie est de 12,2 ans inférieure à celle du citoyen canadien moyen, où la mortalité infantile est trois fois plus élevée qu'au Canada, et le taux de mortalité dû aux maladies respiratoires, aux cancers, aux accidents, aux suicides et aux automutilations, le double de celui du Canada. Pour la période 1999-2003, le taux de mortalité par suicide et par automutilation au Canada était de 9,7 pour 100000 comparativement à

* Respectivement chercheure principale, CCK Research Associates, Ottawa; chercheur associé au groupe de recherche The Impact and Benefit Agreement (IBA) Research Network professeur associé, département de géographie, Université de Guelph, Ontario.
107,3 pour 100000 dans le territoire Inuit Nunangat (Inuit Tapiriit Kanatami 2010). Le taux de tuberculose chez les Inuits demeure le plus élevé dans le monde développé (114,3 pour 100 000) et il est 23 fois plus élevé que parmi la population canadienne.

Bien que les conditions économiques - et plus particulièrement les conditions de vie - expliquent en partie cet état de fait, les raisons de cette disparité en santé demeurent nombreuses et complexes. Les communautés inuites sont aux prises avec un manque constant de maisons, ce qui a provoqué une crise résidentielle en 2006. Selon Statistique Canada (2008: 26), $49 \%$ de la population inuite du Nunavik vivait alors dans des maisons surpeuplées, en comparaison de $3 \%$ pour le reste du Canada. En plus d'augmenter les problèmes sociaux et de santé déjà existants dans ces populations, il est reconnu que vivre dans des maisons surpeuplées augmente le risque d'exposition aux maladies infectieuses, particulièrement les maladies respiratoires, les otites et la tuberculose chez les enfants (Conference Board du Canada 2010; Egeland, Faraj et Osborne 2010; Kovesi et al. 2007). De telles conditions ont 


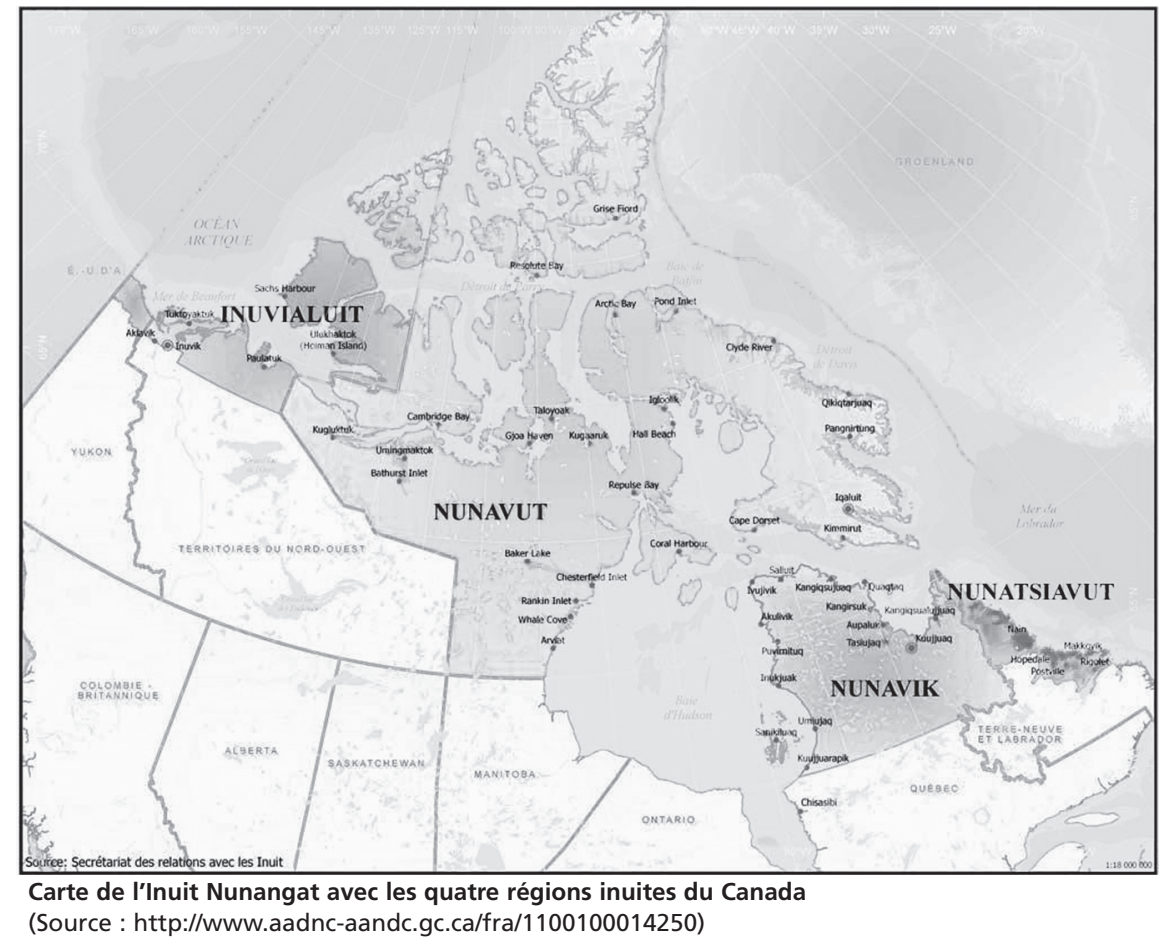

mené à une demande accrue de fonds afin d'apporter des solutions au surpeuplement des logements, soit par des paiements de transfert du fédéral, soit en développant l'économie.

Cette dernière possibilité a reçu une attention grandissante grâce aux nombreuses activités d'exploration et d'exploitation minière dans le Nord canadien. Dans le Nord québécois par exemple, onze grands projets miniers sont en développement, dont deux au Nunavik. Au Nunavut, des activités similaires sont en cours, et les attentes pour des occasions d'affaires et pour l'amélioration du bien-être des communautés sont grandes. Une partie de cet enthousiasme provient des ententes entre les promoteurs de projets miniers et les autorités régionales inuites afin d'atténuer les impacts des activités minières et d'offrir des bénéfices tangibles aux communautés locales; ces ententes sont communément appelées Ententes sur les répercussions et les avantages (ERA ${ }^{2}$ ). Ces ententes sont généralement vues comme étant d'excellents outils pour promouvoir le développement économique des communautés et améliorer certaines de leurs conditions socioéconomiques, plus particulièrement l'accroissement du revenu. Toutefois il n'est pas toujours évident que les communautés ainsi enrichies soient dans un meilleur état quant à leur santé et à leur bien-être. Plus frustrant encore, seulement quelques ERA mentionnent de façon explicite la santé et le bien-être communautaire. Or, ces ententes sont plutôt conçues de manière à générer des bénéfices (financiers) à partir desquels on présume l'amélioration de la santé et du bien-être des communautés.

Cette incapacité à inclure le bien-être des communautés dans les ERA coincide avec l'absence de recherche sur le sujet dans le milieu universitaire. Des chercheurs ont examiné plusieurs aspects de ces ententes tels que la façon de négocier de manière plus efficace (Gibson et O'Faircheallaigh 2010), comment les ERA individuelles diffèrent les unes des autres (Kennett 1999), à quel moment on arrive à des ERA avantageuses et à quel moment elles achoppent (Prno 2007), et comment les ERA devraient contribuer de la meilleure manière possible au développement des communautés (Siebenmorgen 2009). Cependant, moins d'efforts ont été mis à comprendre comment les ERA affectent la santé et le bien-être des communautés - ce qui a pourtant été noté comme étant une priorité dans la recherche (Knotsch et Warda 2009). En étudiant de plus près les ERA signées avec les organisations inuites du Canada, le présent article réitère une demande pour ce type de recherche et suggère qu'entretemps les négociateurs considèrent plus explicitement les questions de santé communautaire dans les futures ententes. Nous partageons une vision positive des ERA comme étant un outil essentiel en vue de diminuer les répercussions négatives des développements miniers chez les populations autochtones et de partager les bénéfices. Toutefois, nous espérons faire prendre conscience de la pertinence d'inclure dans de telles ententes un volet concernant la santé individuelle et le bien-être des communautés.

\section{HistoriQue}

Les Ententes sur les répercussions et les avantages (ERA) sont généralement des ententes confidentielles négociées dans le cadre d'un projet spécifique de développement d'une ressource entre un promoteur et des groupes autochtones/organisations régionales et/ou les gouvernements; parfois, un gouvernement territorial ${ }^{3}$ peut aussi être signataire. Au Canada, les ERA se sont développées comme des ententes privées complémentaires aux permis émis par les agences gouvernementales dans le contexte d'évaluations environnementales (Galbraith et al. 2007). En effet, un des objectifs clés d'une ERA est de rassurer les communautés quant au fait que les répercussions d'un développement minier et de son exploitation - plus 
particulièrement celles qui n'ont pas été prévues durant l'évaluation environnementale - seront surveillées et atténuées. Un deuxième objectif crucial est de rassurer les communautés directement touchées quant au fait qu'elles récolteront des avantages de ce développement - attitude qui contraste avec leur expérience passée, qui n'est pas si lointaine. La force d'une ERA repose en partie sur son pouvoir légal, particulièrement pour les signataires autochtones. Loin d'être parfaites, ces ententes sont tout de même reconnues comme étant de précieux outils.

Au Canada, l'élaboration d'ententes dans le secteur minier est omniprésente. Le ministère des Ressources naturelles tient à jour une cartographie des projets miniers sur le sol canadien en y indiquant les ententes avec les communautés autochtones ou les gouvernements (voir RNCAN s.d.[b]). Sa mise à jour de 2010 indique un total de 171 ententes et lettres d'intention, dont 56 ententes, signées entre des compagnies minières et des organisations autochtones (www.impactandbenefit.com). Aujourd'hui, l'industrie accepte les ERA comme allant de soi et c'est la façon de faire dans le nord du Canada (Lapierre et Bradshaw 2008; Scales 2010). Dans les juridictions canadiennes où les ERA relèvent de la bonne volonté, les entreprises en sont venues à les signer afin d'obtenir une autorisation sociale pour s'implanter dans les territoires traditionnellement occupés ou utilisés par les communautés autochtones. Dans les régions où des accords sur les revendications territoriales ont été signés, par exemple au Nunavut ou au Nunatsiavut, les ERA sont obligatoires par la loi.

Le titre des ententes varie mais le contenu d'une ERA inclut des bénéfices financiers directs et indirects, des possibilités d'emploi et d'éducation, des mesures pour le développement culturel local et la protection de l'environnement (Kennett 1999; Sosa et Keenan 2001). La réhabilitation des sites et la préservation d'habitats de valeur significative ou d'autres sites naturels sont, pour les communautés signataires, des dispositions importantes quant à la protection et à la conservation de l'environnement (Gibson et Klinck 2005; Galbraith et al. 2007; Fidler et Hitch 2007). Les bénéfices financiers directs incluent le partage des profits et des versements comptants, en plus de fonds de compensation pour atténuer les répercussions du développement minier (Kennett 1999; Hodge et Killam 2003). Les bénéfices indirects versés aux communautés peuvent inclure une politique d'attribution de contrats sur une base préférentielle aux entrepreneurs locaux pour les besoins des minières durant la construction et les activités d'opérations (Kennett 1999; O'Faircheallaigh 2006). Dans leur IBA Community Toolkit, Gibson et O'Faircheallaigh (2010) proposent des conseils judicieux aux signataires autochtones, à savoir comment évaluer les diverses provisions économique - par ex. les actions, les redevances basées sur les bénéfices ou sur la valeur, ou encore le volume de la production - et les paiements fixes

Les cibles locales d'emploi et les subventions accordées pour la formation sont au cœur des négociations dans la plupart des ERA (Kennett 1999; Sosa et Keenan 2001) et sont également parmi les avantages les plus importants de ces ententes (voir Kinross Gold Corporation 2007; DeBeers Canada 2009). À travers le Canada, l'emploi des autochtones dans le secteur minier est en hausse depuis les dix dernières années. Selon le recensement de 2006, les autochtones représentent 7,5\% du total de la maind'œuvre dans le secteur minier comparativement à 5,1\% en 2001 (RNCAN 2009). Cependant, l'emploi n'est qu'une partie d'un système devant être mis en place afin de permettre le bien-être des membres d'une communauté. Des fonds peuvent aussi être mis de côté pour des « avantages souples» (soft benefits) tels que des activités socioculturelles et le développement d'infrastructures (Sosa et Keenan 2001).

Nous pouvons observer des centres d'intérêt différents entre les ententes signées. Par exemple, l'entente Raglan insiste fortement sur la protection de l'environnement (Telewiak 2001). Lentente de Voisey's Bay porte une attention particulière aux activités traditionnelles et la formation, et la plus récente entente, l'entente de Meadowbank, se concentre sur l'emploi et la formation régionale. Bien que, comme le soutiennent Siebenmorgen et Bradshaw (2011), il y ait une variété illimitée d'éléments qui pourraient faire partie d'une ERA, en pratique toutefois ces éléments ont été largement limités aux intérêts économiques. En outre, dans certaines communautés il n'y a pas nécessairement unanimité, et plusieurs sont d'avis de ne pas avoir été justement compensés. Par exemple, les chasseurs et pêcheurs traditionnels pouvant être directement affectés par les répercussions du développement ont reçu peu de compensations et bénéficient encore moins de ce développement (ONSA 2008; Siebenmorgen 2009). Puisque chaque ERA est élaborée et négociée dans le cadre d'un projet spécifique ayant ses propres besoins, répercussions et solutions, il y a un énorme potentiel pour que ces ententes deviennent d'importants véhicules de développement économique et social pour les communautés (Siebenmorgen et Bradshaw 2011).

\section{LE RÔLE GRANDISSANT DES ERA DANS LES RÉGIONS INUITES}

Les Inuits du Canada ont conclu des revendications territoriales dans quatre régions géographiques: le Nunavik, comprenant les terres et les régions marines dans la partie nord de la province de Québec; le Nunatsiavut, comprenant la côte et la région nordique du Labrador; le territoire du Nunavut; et la « région désignée 


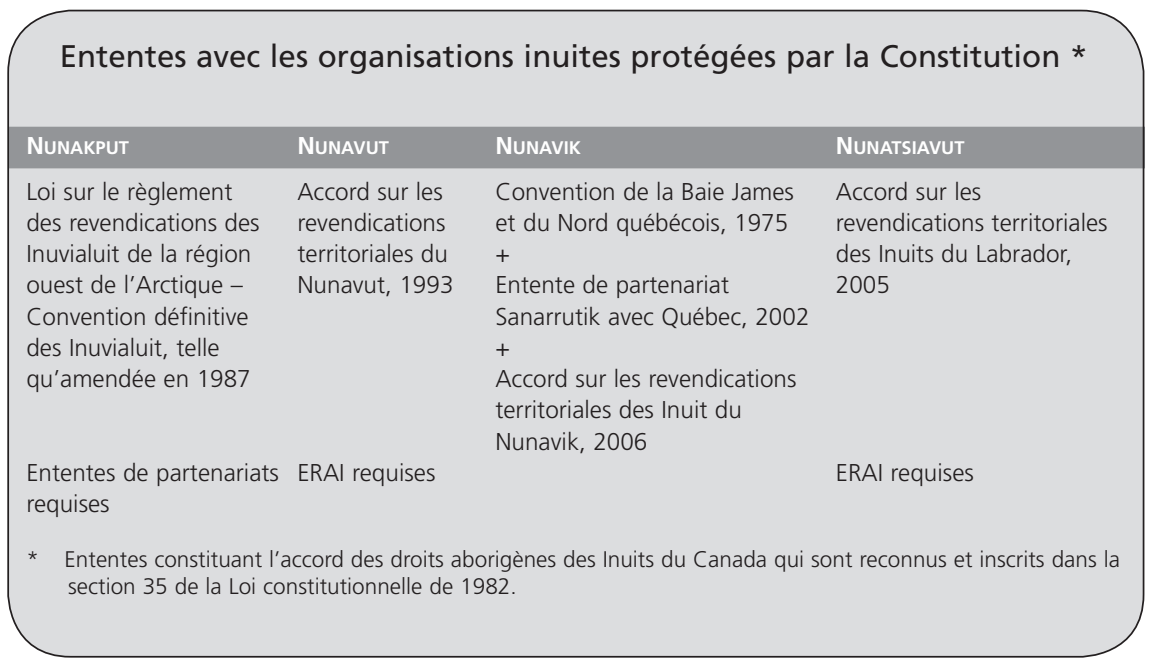

gestion des ressources fauniques de la région marine du Nunavik.

En plus, le gouvernement du Québec a signé une entente de partenariat d'une durée de vingtcinq ans avec les Inuits du Nunavik afin d'accélérer le développement économique au Nunavik. Par l'entente Sanarrutik, le gouvernement du Québec s'engage à faciliter et à encourager la signature d'ententes entre la société Makivik et les compagnies minières en ce qui a trait à la restauration des sites miniers et aux retombées financières, et pour l'attribution de contrats de service (AADNC 2007).

des Inuvialuit». Cette dernière comprend plusieurs sous-régions : la mer de Beaufort, le delta du Mackenzie, la partie septentrionale du Yukon, la partie nord-ouest des Territoires du Nord-Ouest et l'archipel arctique canadien. Ensemble, ces quatre régions inuites forment l'Inuit Nunangat. Bien que ces quatre régions aient des règles pour promouvoir l'établissement de ERA (voir tableau), il existe de subtiles différences d'une région à l'autre quant à leurs intérêts et à leur approche dans les négociations des ententes. Nous allons donc étudier les règles du développement minier et l'usage des ERA dans chacune des quatre régions, et le cas échéant, nous porterons une attention particulière à certains projets de développement pouvant servir de modèle.

\section{Le Nunavik}

Le Nunavik est la patrie des Nunavimmiuts du nord du Québec, dont les intérêts sont représentés par la société Makivik. Au Nunavik, deux régimes constitutionnels s'ajoutent aux lois sur le développement minier : la Convention de la Baie James et du Nord québécois (1975) -complémentaire aux lois du Québec sur le territoire du Nunavik -, et l'Accord sur les revendications territoriales des Inuit du Nunavik - qui est complémentaire aux lois du Nunavut sur les îles extra-côtières et les régions marines. Malgré le fait que l'Accord ne contient pas de volet pour une autonomie gouvernementale ${ }^{4}$, il est tout de même enchâssé dans une loi pour la protection des terres et pour le droit aux ressources afin de promouvoir le développement économique et social. À cette fin, les Inuits du Nunavik pourront recevoir des redevances annuelles du gouvernement du Canada issues de l'exploitation éventuelle des ressources dans la région marine du Nunavik. Les Nunavimmiuts peuvent aussi participer à la gestion de la ressource faunique dans la région extracôtière par l'intermédiaire du Conseil de
À ce jour, l'entente la plus significative signée par la société Makivik fut l'entente Raglan (1995) négociée lors de la mise en œuvre de la mine Raglan. L'exploration de la mine Raglan, située dans le nord du Nunavik à environ 100 kilomètres de la baie Déception, a débuté en 1957 et elle a amorcé sa production en 1998, on y extrait du nickel, du cuivre, du platine et du cobalt. Le propriétaire original, Falconbridge Ltd, a embauché à l'époque un agent de liaison pour faire le lien avec les deux communautés voisines, Salluit et Kangiqsujuaq; Falconbridge s'engageait alors à offrir $20 \%$ des postes à des Inuits. Depuis 2006, la mine Raglan est propriété de la compagnie suisse Xstrata. Les statistiques de cette dernière en 2008 indiquent que, des 500 travailleurs de la mine, 16 \% étaient des Inuits. Les niveaux d'emploi sont l'un des nombreux éléments de base de l'entente Raglan signée en 1995 entre Falconbridge et la société Makivik. L'entente prévoit des mesures de participation aux bénéfices et des versements à un fonds fiduciaire sur une période de dix-huit ans, de même que des initiatives de formation et autres plans de développement économique. De plus, l'entente garantit que les emplois et les contrats seront accordés de préférence aux Inuits et entreprises inuites des environs qui sont qualifiés pour faire le travail. Toutefois cela n'était pas suffisant, et les discussions entre Falconbridge et les deux communautés n'ont pas été fructueuses. Les communautés n'étaient pas satisfaites de l'approche de Falconbridge et elles ont demandé à la société Makivik d'intervenir en leur nom. Les préoccupations des Inuits étaient plutôt centrées sur les questions d'environnement, d'emploi, d'utilisation traditionnelle $\mathrm{du}$ territoire, tandis que la Compagnie voulait surtout contribuer aux infrastructures locales.

Lentente Raglan offre plusieurs avantages aux Inuits. Par exemple, le «comité Raglan» constitué de six 
membres dont la moitié doivent être inuits, est le principal porte-parole pour les communications entre la Compagnie et les communautés et il permet un cadre efficace à la coopération. L'Entente stipule aussi la priorité d'emploi et la priorité de contrats aux entreprises inuites, des compensations financières, le partage des profits et, enfin, elle offre un processus d'arbitrage. L'ERA comporte des clauses quant aux répercussions environnementales (qualité de l'eau, habitat des poissons, etc.). Malgré le fait que l'entente Raglan a été louangée pour sa volonté de créer un esprit de coopération et de résolution des problèmes, et pour l'encouragement d'une éducation formelle, des préoccupations ont tout de même été exprimées. Par exemple, l'ERA omet la surveillance des répercussions sociales (monoparentalité, enfants négligés, soin de longue durée des enfants, abus de substances, discrimination raciale, taux d'avancement plus lents pour les employés inuits, etc.). De plus, les compensations monétaires et contractuelles n'ont pas apporté les développements escomptés. Ces préoccupations pourront être prises en considération au moment où Xstrata Nickel cherchera à accroître ses opérations pour devenir l'une des plus grandes minières de la planète et devra, par conséquent, revoir les ententes existantes. Sans aucun doute, la mine Raglan bénéficie aux communautés environnantes par le biais des emplois, des contrats de services et par le programme annuel de partage des profits; il est toutefois moins évident que la santé de ces communautés se soit améliorée depuis le début des activités minières et la signature de l'ERA.

\section{Le Nunavut}

Le Nunavut a été établi en 1999 par l'Accord sur les revendications territoriales du Nunavut. Trois associations inuites régionales représentent les intérêts des bénéficiaires: l'Association inuite Kitikmeot, l'Association inuite Kivalliq et l'Association inuite Qikiqtani. Dans un contexte de développement minier, cela signifie qu'elles sont mandatées pour négocier avec les promoteurs au nom des communautés. Ces négociations sont inscrites dans l'Accord sur les revendications territoriales du Nunavut qui stipule qu'une Entente sur les répercussions et les avantages pour les Inuits (ERAI) doit être conclue avant qu'un développement minier majeur puisse aller de l'avant ${ }^{5}$. Afin d'éviter des délais et s'assurer que les avantages d'une ERAI « ne doivent pas imposer un fardeau excessif au promoteur et ainsi miner la viabilité du projet» (Accord sur les revendications territoriales du Nunavut, art. 26.3.3[d]), un échéancier pour les négociations a été établi avec des options d'arbitrage volontaires et obligatoires lorsque des conflits surgissent. Toutes les parties se doivent de négocier une ERAI de bonne foi.
Au Nunavut, les dispositions d'une ERA consolident l'article 25 de l'entente sur les revendications territoriales du Nunavut, qui stipule que les Inuits recevront une part des redevances prélevées sur les ressources exploitées sur les terres de la Couronne. Les redevances -dont le taux de rendement minimum est de $12 \%$ des profits - sont déposées dans la Fiducie du Nunavut (Nunavut Trust). La Nunavut Tunngavik Inc. (NTI), organisation inuite chargée de s'assurer que l'entente sur les revendications territoriales du Nunavut est respectée, a délégué le rôle de négociateurs d'une ERAI aux organisations inuites désignées (OID) de la région. Deux projets miniers majeurs - l'un en opération, l'autre à l'étude - ont été soumis à ce processus de négociation.

Le premier projet, la mine d'or Meadowbank située à 70 kilomètres au nord de Baker Lake (110 km via un accès routier toute saison), est en opération depuis mars 2010. Le permis fut émis en 2006, l'année même de la signature d'une ERAI entre la Cumberland Resources Ltd. et l'Association inuite Kivalliq (AIA). Après l'achat de l'entreprise par Agnico-Eagle, une nouvelle ERAI fut négociée et entérinée en octobre 2011. En plus des redevances à l'AIA, les deux ententes contiennent des dispositions sur la formation et l'éducation, le soutien au développement économique des Inuits par la dotation préférentielle de contrats, et elles procurent des subventions pour les ressources fauniques. Un des éléments clés dans la négociation des ententes est la participation accrue de la main-d'œuvre inuite à la mine, particulièrement pour les Inuits de la région de Kivalliq. Quelques progrès ont été accomplis en ce sens; à la fin de l'année 2011, des 631 employés à Meadowbank, 231 provenaient du Nunavut (37\%), dont 128 étaient de Baker Lake (Kivalliq SEMC 2011). Dans cette communauté, on remarque une amélioration évidente de la richesse en dénombrant un nombre croissant de véhicules toutterrain ou le nombre d'individus s'envolant vers Winnipeg pour le magasinage. Ce qui est moins sûr, c'est de savoir si la santé communautaire s'est améliorée.

Le second projet qui retient l'attention au Nunavut, bien qu'il soit encore à l'étude, est le projet de mine de fer de Mary River, propriété de la société Baffinland Iron Mines Corp. Le site minier proposé, situé au nord de l'île de Baffin à $106 \mathrm{~km}$ au sud de Pond Inlet, a pour but de fournir du fer aux marchés européens et requiert la construction d'un port en eaux profondes à Steensby Inlet. Les négociations pour une ERAI sont déjà en cours avec l'Association inuite Qikiqtani (AIQ), l'organisation inuite désignée pour cette région. Un seul employé à temps plein est en poste à l'AIQ afin de participer aux négociations et de transmettre les préoccupations et les intérêts des membres de la communauté. 


\section{Le NunakPut ${ }^{6}$}

Dans la région septentrionale des Territoires du NordOuest, le regroupement des associations inuvialuites (Inuvialuit Corporate Group) - composé de la Société régionale Inuvialuit (Inuvialuit Regional Corporation) et de ses filiales - représente les communautés inuites. La Convention définitive des Inuvialuit signée en 1984 et amendée en 1987, a été la troisième entente exhaustive sur les revendications territoriales au Canada. Cette entente insiste sur la préservation de l'identité culturelle des Inuvialuits et de leurs valeurs dans une société nordique en pleine évolution, sur une participation significative dans l'économie et la société, tant nordique que nationale, ainsi que sur la protection et la préservation de l'environnement, de la faune arctique et de la productivité biologique. LEntente finale des Inuvialuits requiert que les ERA, comme " entente de participation », soient négociées lorsque l'usage du sol est plus que temporaire et comporte des garanties de paiement par le promoteur pour une compensation juste pour tous dommages causés aux terres des Inuvialuits. Nous pouvons également y inclure un loyer approprié pour l'utilisation du territoire (n'incluant aucune redevance) et des termes et conditions spécifiques en respectant la nature et l'ampleur de l'utilisation du territoire pour lesquels l'accès est demandé. Les conditions de l'entente peuvent également prévoir :

- les frais engagés par l'ATI [Administration des terres des Inuvialuit] pour l'inspection de l'emplacement des travaux et la nature et l'étendue de l'inspection;

- le renouvellement des ressources fauniques, les mesures d'indemnisation et de redressement;

- l'emploi, les contrats de service et d'approvisionnement;

- l'enseignement et la formation;

- les titres de participation et les divers types d'avantages liés à la participation.

(Convention définitive des Inuvialuit, Section 10.3)

De plus, la Convention définitive des Inuvialuit limite le type de développement qui serait proposé dans la partie est de North Slope. Quel que soit le type de développement, incluant les forages de pétrole ou de gaz, il est sujet à des études environnementales qui prévoient la participation des Inuvialuits, des représentants du territoire et ceux du gouvernement fédéral.

\section{Le Nunatsiavut}

Le Nunatsiavut comprend la région visée par le règlement des Inuits du Labrador. Le gouvernement du Nunatsiavut (auparavant l'Association des Inuit du Labrador) représente les Inuits et les Kablunangajuits (non inuits).
LAccord sur les revendications territoriales des Inuit du Labrador (2005) spécifie que les ERAI doivent être négociées entre le gouvernement du Nunatsiavut et les promoteurs avant qu'un projet puisse obtenir son aval dans les terres des Inuits du Labrador et avant que des développements majeurs aient lieu dans le territoire situé à l'extérieur des terres des Inuits du Labrador (Accord sur les revendications territoriales des Inuit du Labrador 2005, chap. 7, partie 7.7). Le gouvernement du Nunatsiavut peut percevoir $25 \%$ des recettes provinciales provenant des projets d'exploitation des ressources souterraines des terres des Inuits du Labrador. Dans la région située à l'extérieur des terres des Inuits du Labrador, le gouvernement du Nunatsiavut recevra $50 \%$ de la première tranche de deux millions de dollars et $5 \%$ des recettes provinciales additionnelles provenant des projets d'exploitation du sous-sol. Dans la région située à l'extérieur des terres des Inuits du Labrador, les revenus perçus sur les ressources souterraines seront limités à un montant qui, s'il était distribué également entre les Inuits du Labrador, se calculerait sur la moyenne des revenus par personne des Inuits du Labrador et équivaudrait à la moyenne canadienne du revenu par personne (Nunatsiavut Government, s.d.).

Les ententes sur les répercussions et les avantages pour les Inuit (ERAI) devront être négociées entre le promoteur et les Inuits du Labrador et seront obligatoires pour tout développement sur les terres des Inuits du Labrador et pour tout développement majeur dans la région à l'extérieur de ces mêmes terres. Un développement est considéré comme majeur lorsque le capital investi est d'au moins quarante millions de dollars ou de cent cinquante années-personnes par périodes de cinq ans (Labrador Inuit Association, s.d.). Le projet de la mine de nickel de Voisey's Bay, qui depuis 2005 produit du nickel, du cuivre et du cobalt pour son propriétaire, Vale-Inco, fait partie de cette catégorie. Cette mine est située au Labrador, à $35 \mathrm{~km}$ au sud-ouest de la ville de Nain et à $350 \mathrm{~km}$ au nord de Happy Valley-Goose Bay. En raison du chevauchement du site minier sur les territoires de la nation innue et ceux des Inuits du Nunatsiavut, des ententes séparées ont été négociées avec la compagnie Voisey's Bay Nickel, une filiale d'Inco ltée. L'ERAI signée avec les Inuits du Labrador leur donne préséance quant à la formation, l'emploi et les occasions d'affaires reliées au projet de la baie de Voisey. Les autochtones et les gouvernements seront consultés avant tout développement minier, toute exploration, et sur toutes les mesures pour préserver, protéger et réhabiliter l'environnement en relation avec le projet de la baie de Voisey. De plus, l'ERAI offre un partage de $5 \%$ des revenus pour l'Association des Inuit du Labrador. L'ERA signée par la nation innue - conclue après des années de négociations - s'assure que 
le gouvernement innu (Sheshatshiu et Natuashish) reçoive de la province de Terre-Neuve-et-Labrador $5 \%$ de tous les revenus provenant du projet minier de la baie de Voisey.

Les accords signés avec la mine Voisey's Bay ont permis d'atteindre un taux d'emploi de $54 \%$ chez les autochtones sur un total de 500 emplois sur le site minier (RNCAN, s.d.[a]), ce qui constitue le niveau le plus élevé de la participation des autochtones dans le secteur minier au Canada. D'autres dispositions des ERAI - telles que le développement des affaires locales, totalisant plus de 515 millions de dollars en contrats durant la phase de construction, la création d'un fonds du patrimoine, la mise sur pied d'un bureau pour le personnel de l'ERAI, l'éducation continue et la formation offerte via le programme du centre de développement professionnel -font que les communautés concernées obtiennent des avantages significatifs. Le ministère des Ressources naturelles a même qualifié la mine Voisey's Bay de "modèle de participation et de progression rapide des groupes autochtones dans l'industrie minière » (ibid.). Malgré ces éloges, une analyse systématique des communautés affectées par le projet doit être complétée afin d'évaluer si les avantages contribuent à améliorer la santé et le bien-être tant des communautés inuites et innues que des familles et des individus.

\section{FAIRE EN SORTE QUE LES ERA AMÉLIORENT LE BIEN-ÊTRE DES COMMUNAUTÉS}

Pour les communautés situées à proximité des activités minières, particulièrement celles où l'état de santé des populations accuse un retard par rapport à l'ensemble du Canada, il est attendu que les exploitations minières, surtout celles accompagnées d'ERA, apportent des avantages à la communauté et améliorent les conditions de santé de sa population. Toutefois, il devient de plus en plus évident que les ERA génèrent de l'argent et des emplois mais qu'un développement économique ne génère pas nécessairement et automatiquement des communautés plus en santé. Bien que la mise en œuvre d'une ERA y joue clairement un rôle, il est aussi évident que les récentes ERA, dans les régions inuites comme ailleurs, privilégient nettement les bénéfices économiques tels que les compensations financières, les contrats et les emplois plutôt que la santé et le bien-être des communautés. En d'autres termes, les bénéfices économiques prévalent au détriment des investissements dans des programmes sociaux et des programmes de santé (Knotsch et Warda 2009, Fidler et Hitch 2007, O'Faircheallaigh 1999). Les participants inuits sont d'accord pour que les questions sociales et le secteur de la santé soient prioritaires lors des négociations des ERA. Toutefois les structures de négociation et l'accent mis sur les bénéfices économiques et fiscaux deviennent souvent des barrières à des considérations plus réfléchies quant aux préoccupations sociales et à la santé (Knotsch et Warda 2009). Malheureusement la nature "privée » des ERA empêche souvent la réflexion et le débat sur les problèmes de santé (Gibson et Klinck 2005; Prno et Bradshaw 2008)

Une des faiblesses propres aux ERA est le fait qu'elles reposent sur la négociation et qu'il est difficile de négocier sur des questions de bien-être. Les pratiques et les attitudes habituelles dans les négociations des ERA - par exemple le fait que les emplois sont la priorité - sont dues à un état d'esprit orienté vers des résultats économiques directs et généralement quantifiables. Le manque de définition claire du bien-être rend également plus difficile de négocier dans les ententes des objectifs à long terme et un soutien à la santé. Une autre faiblesse - et à la fois un défi - est la possibilité de conflit face aux politiques et aux services gouvernementaux lors de l'examen des dispositions concernant les questions sociales et la santé. À ce titre, les services fédéraux et ceux d'autres juridictions pourraient être affectés si au même moment les clauses d'une entente privée offraient des services similaires. Il est compréhensible de s'inquiéter que les programmes gouvernementaux, déjà limités, puissent être réduits si des services pour la santé ou l'éducation, ou des services sociaux, devaient être offerts par des ententes corporatives (Knotsch et Warda 2009).

Nonobstant ces questions, il est possible d'inclure le bien-être des communautés et des activités connexes dans la négociation des ERA. Une des approches est d'être plus attentifs aux préoccupations touchant la santé durant le processus d'évaluation environnementale et d'élaborer une définition plus élargie de la santé. L'étude de Noble et Bronson (2005) portant sur le processus d'évaluation environnementale de la mine Voisey's Bay montre bien que la définition de la santé était trop restrictive. Une autre avenue permettant d'intégrer dans les ERA des mesures pouvant aider à une meilleure santé est d'abord d'en reconnaître les déterminants sociaux, c'està-dire les facteurs qui influencent les résultats de santé mais qui sont de nature sociale plutôt que médicale. Dans le contexte autochtone par exemple, les chercheurs ont indiqué le colonialisme, le racisme, l'exclusion sociale et l'autodétermination comme variables qui influencent fortement la santé (Richmond 2009; Richmond et Ross 2009; Loppie Reading et Wien 2009).

Dans le contexte particulier des mines, les chercheurs ont reconnu le rôle critique de facteurs non médicaux, tels le logement, l'éducation, l'exclusion sociale et la discrimination, sur la santé des individus et des populations (Evans et Stoddart 1990; Marmot 2001; Williams 2003; Marmot et al. 2008). Des études ont permis d'identifier 
les impacts sur la santé qui incluent la marginalisation des femmes au foyer comme source de stress (Sharma et Rees 2007), ainsi que l'accroissement d'abus de substances illicites et des pratiques sexuelles non sécuritaires (Desmond et al. 2005; Campbell 1997; Steen et al. 2000; Palmer et al. 2002). Ces études ont aussi exploré les déterminants sociaux, psychologiques et structurels de la santé sexuelle (Campbell 2000) dans les communautés minières, pétrolières et gazières (Goldenberg et al. 2008). On remarque dans ces communautés un taux plus élevé de violence conjugale, de maladie mentale, d'exploitation sexuelle, d'abus de substances et de jeux de hasard (Desmond et al. 2005; Gibson et Klink 2005; Marmot 2001). Sharma et Rees (2007) ont examiné l'incidence de désordres psychologiques chez les conjointes de mineurs dans des communautés minières isolées d'Australie, identifiant les horaires de travail, l'isolement des femmes à la maison et la nature épuisante du travail dans une mine comme sources potentielles de stress. Finalement, chez les peuples autochtones, pour qui la terre demeure la mère nourricière et le renouveau culturel, perturber l'usage traditionnelle de la terre par le développement minier peut avoir des répercussions sur la santé, sur l'estime de soi, sur l'organisation et l'interaction communautaires, ainsi que sur le sentiment d'appartenance dans le monde moderne (Environmental Studies Research Fund 2008).

En reconnaissant ces déterminants sociaux de la santé, les négociateurs d'une ERA peuvent élargir leur champ d'action par une nouvelle façon de voir. Ils peuvent aussi bénéficier d'une meilleure connaissance des problèmes associés aux salaires élevés, aux horaires particuliers et au travail en milieu éloigné que causent les projets miniers. C'est bien connu que les mineurs souffrent de fatigue extrême, de stress mental, d'anxiété, de dépression et même d'idées suicidaires. La toxicomanie et les comportements à risque ont aussi été identifiés comme étant des problèmes courants dans le travail à horaire prolongé et à salaire élevé (par ex. Brubacher 2002). Des répercussions moins significatives, tels un changement de diète ou la perturbation de la vie familiale, peuvent causer des problèmes non seulement pour les travailleurs mais aussi pour leur famille, incluant des répercussions sur la fréquentation scolaire (Gibson et Klinck 2005).

Finalement, bien que des études sur les négociations des ententes puissent éventuellement mener à des ERA mieux ciblées en ce qui a trait à la santé, une mise en œuvre plus efficace doit être faite pour assurer aux communautés des gains véritables dans ce secteur, car la relation entre une ERA et le bien-être des communautés repose largement sur la mise en application de l'entente.
En résumé, les ententes sur les répercussions et les avantages (ERA) offrent aux communautés la possibilité d'identifier les avantages possibles pour leur communauté, de formuler des buts et des perspectives à envisager et de déterminer le moment où les bénéfices pourront être réalisés. Une approche de santé communautaire au début du processus de négociation peut aider non seulement à développer une vision à long terme, mais aussi à influencer le contenu d'une ERA pour y inclure des clauses sur la santé des individus et des communautés. Les possibilités des ERA sont immenses et incluent l'option d'ouvrir le processus de négociation afin de permettre à d'autres d'apprendre de ces pratiques, de parvenir à la confiance mutuelle, à la compréhension et au respect, et enfin, de s'assurer que la santé et le bienêtre des communautés soient la priorité absolue.

\section{Notes}

1. L'Inuit Nunangat comprend les quatre régions inuites du Canada : Inuvialuit, Nunavut, Nunavik et Nunatsiavut. Voir http://www. itk.ca/publication/maps-inuit-nunangat-inuit-regions-Canada.

2. Au Nunavut, elles sont connues comme Ententes sur les répercussions et les avantages pour les Inuits (ERAI).

3. Les gouvernements provinciaux, malgré leur rôle de facilitateurs des ERA, ne sont généralement pas signataires.

4. http://www.ainc-inac.gc.ca/nr/prs/s-d2007/2-2956-faq-eng.asp

5. Il est stipulé que, dans la région du Nunavut, tant la Couronne que le secteur privé sont tenus d'obtenir une ERAI pour l'exploitation de l'eau ou le développement des ressources qui « entraîne, dans la région du Nunavut, sur une période de cinq ans, soit l'emploi de plus de 200 années-personnes, soit l'engagement de dépenses en immobilisations supérieures à trente-cinq millions de dollars (35000 000 \$), en dollars constants de $1986 »$. (Accord sur les revendications territoriales du Nunavut, art. 26.1.1[b], voir <http://publications. gc.ca/collections/Collection/R32-134-1993F.pdf $>$ ).

6. Le Nunakput est aussi connu sous le nom de « région désignée des Inuvialuit ». Les Inuvialuits font référence à leurs terres comme étant le Nunakput, situé dans la circonscription électorale septentrionale des Territoires du Nord-Ouest. La circonscription électorale du Nunakput comprend les communautés de Paulatuk, Sachs Harbour, Tuktoyaktuk et Ulukhaktok. La Convention définitive des Inuvialuit inclut aussi les droits sur le territoire d'Inuvik.

\section{Ententes et ouvrages cités}

\section{Ententes}

Accord entre les Inuit de la région du Nunavut et sa Majesté la Reine du Chef du Canada. Signé à Iqaluit (T.-N.-O.), le 25 mai 1993. Voir <http://publications.gc.ca/collections/Collection/R32-1341993F.pdf>, (consulté le 20 février 2012).

Accord entre les Inuit du Nunavik et sa Majesté la Reine du Chef du Canada sur les revendications territoriales des Inuit du Nunavik. Signé à Kuujjuaq, le $1^{\text {er }}$ décembre 2006. Voir <http://publications.gc.ca/collections/collection_2007/inac-ainc/R2-4542006F.pdf>, (consulté le 20 février 2012). 
Accord sur les revendications territoriales entre les Inuit du Labrador et Sa Majesté la Reine du Chef de Terre-Neuve-et-Labrador et Sa Majesté la Reine du Chef du Canada. Signé à Nain, NFLD, le 22 janvier 2005. Voir <http://www.aadnc-aandc.gc.ca/fra/1293647036287>, (consulté le 20 février 2012).

Convention de la Baie James et du Nord québécois et conventions complémentaires [version de 1998]. Signée à Québec, le 11 novembre 1993. Voir <http://epe.lac-bac.gc.ca/100/200/301/inac-ainc/ james_bay-f/jbnq_f.pdf>, (consulté le 20 février 2012).

Convention définitive des Inuvialuit - Revendication de l'Arctique de l'Ouest, telle qu'amendée en 1987. Signée à Tuktoyaktuk, le 5 juin 1984. Version définitive: <http://www.collectionscanada. gc.ca/webarchives/20071125082144/http://www.ainc-inac. gc.ca/pr/agr/inu/wesar_f.pdf> et les modifications : <http:// www.aadnc-aandc.gc.ca/fra/1100100031048>, (consulté le 20 février 2012)

\section{Documentation}

AADNC (Affaires autochtones et Développement du Nord Canada), 2007: Accord sur les revendications territoriales des Inuit du Nunavik - Foire aux questions. Disponible sur Internet : < http:// www.aadnc-aandc.gc.ca/aiarch/mr/nr/j-a2007/2-2855-faq-fra. asp>, (consulté le 20 février 2012).

ADELSON, N., 2005 : «The Embodiment of Inequity: Health Disparities in Aboriginal Canada ». Canadian Journal of Public Health/Revue canadienne de santé publique 96 : S45-S61.

BRADSHAW, Ben, et Peter SIEBENMORGEN, 2010 : What and when IBAs, MoUs, MEAs, and JVs? Communication à la $5^{\text {th }}$ Annual Learning Together Conference, Vancouver, April 8-9.

BRUBACHER AND ASSOCIATES, 2002 : The Nanisivik Legacy in Arctic Bay: A Socio-Economic Impact Study. Rapport pour le Department of Sustainable Development, Government of Nunavut, Ottawa.

CAMPBELL, C., 1997 : « Migrancy, masculine identities and AIDS: The psychosocial context of HIV transmission on the South African gold mines ». Social Science \& Medicine 45(2) : 273-281.

—, 2000 : "Selling sex in the time of AIDS: The psycho-social context of condom use by sex workers on a Southern African mine ». Social Science E Medicine 50(4) : 479-494

CONFERENCE BOARD DU CANADA, 2010: Sleeping on the couch. Centre for the North. Disponible sur Internet : <www. centreforthenorth.ca/blogs/herethenorth/sleepingonthecouch>, (consulté le11 août 2011).

DEBEERS CANADA, 2009 : Socio-Economic Results Released for Snap Lake Mine. Media Release, May 28th. Disponible sur Internet : $<$ http://www.debeerscanada.com/files_2/documents/2009-0527-DBC-News-Release-Socio-Ec-Report.pdf>, (consulté le 20 février 2012)

DESMOND, N., et al., 2005: «A typology of groups at risk of HIV/STI in a gold mining town in north-western Tanzania ». Social Science \& Medicine 60(8) : 1739-1749.

EGELAND, G.M., N. FARAJ et G. OSBORNE, 2010 : «Cultural, socioeconomic, and health indicators among Inuit preschoolers: Nunavut Inuit Child Health Survey, 2007-2008 ». Rural and Remote Health 10 : 1-13.

ENVIRONMENTAL STUDIES RESEARCH FUND, 2008: Traditional Knowledge Guide for the Inuvialuit Settlement Region, Northwest Territories. Vol. II: Using Traditional Knowledge in Impact Assessments. Environmental Studies Research Funds Report n 153. Calgary, Alberta
EVANS, R. G., et G.L. STODDART, 1990 : « Producing health consuming health care ». Social Science and Medicine 31(12) : 1247.

FIDLER, C., et M. HITCH, 2007 : « Impact and Benefit Agreements: A contentious issue for environmental and Aboriginal justice ». Environments Journal 36(2): 49-69.

GALBRAITH, L., B. BRADSHAW et M.B. RUTHERFORD, 2007 : « Towards a new supraregulatory approach to environmental assessment in Northern Canada ». Impact Assessmentand Project Appraisal 25(1): 27-41

GIBSON, G., et J. KLINCK, 2005: « The Impact of Mining on Aboriginal Communities, Canada's Resilient North». Pimatisiwin 3(1): 115 - 141.

GIBSON, G., et C. O'FAIRCHEALlaigh, 2010 : IBA Community Toolkit. Negotiation and Implantation of Impact and Benefit Agreements. Walter \& Duncan Gordon Foundation, Toronto.

GOLDENBERG, S., J. SHOVELLER, A. OSTRY et M. KOEHOORN, 2008 : « Youth sexual behaviour in a boomtown: Implications for the control of sexually transmitted infections ». Sexually Transmitted Infections 84(3) : 220-223

HITCH, Michael W., 2006 : Impact and Benefit Agreements and the Political Ecology of Mineral Development in Nunavut. Thède de doctorat, département de géographie, Université de Waterloo, Waterloo, Ontario. Disponible sur Internet : <http://uwspace. uwaterloo.ca/bitstream/10012/992/1/mhitch2006.pdf>, (consulté le 20 février 2012)

HODGE, R.A. et R. KILLAM, 2003 : Post mining regeneration best practice review : North American perspective. Preparé par ECUS Environmental Consultancy, University of Sheffield, Sheffield, England et The Eden Project, 7 décembre. Disponible sur Internet : <http://www.anthonyhodge.ca/publications/Post_ Mining_Regeneration.pdf>, consulté le 12 mars 2012).

INUIT TAPIRIIT KANATAMI, 2010 : Health indicators of Inuit Nunangat within the Canadian context 1994-1998 and 1999-2003. Ottawa. Disponible sur Internet: <http://www.itk.ca/sites/default/ files/20100706Health-Indicators-Inuit-Nunangat-EN.pdf>, (consulté le 20 février 2012).

KENNETT, S., 1999: A Guide to Impact and Benefits Agreements. Canadian Institute of Resources Law, Calgary, Alberta.

KINROSS GOLD CORPORATION, 2007 : Corporate Responsibility. Site Internet : <http://www.kinross.com/corporate-responsibility. aspx>, (consulté le 20 février 2012).

KIVALLIQ SEMC, 2011: Kivalliq Socio-Economic Monitoring Committee Annual Report, August 31, 2010. Disponible sur Internet : <http://ftp.nirb.ca/03-monitoring/regional\%20semc/ kivalliq/>, (consulté le 20 février 2012).

KNOTSCH, C., et J. WARDA, 2009 : Impact and Benefit Agreements: A tool for healthy communities? National Aboriginal Health Organization, Ottawa. Disponible sur Internet : <http://www. naho.ca/documents/it/2010_IBA_Report.pdf>, (consulté le 12 mars 2012)

KOVESIE, T., et al. 2007 : "Indoor air quality and the risk of lower respiratory tract infections in young Canadian Inuit children ». Canadian Medical Association Journal 177(2): 155-160

LABRADOR INUIT ASSOCIATION, s.d. : Labrador Inuit Association Perspective. Site Internet : <http://arcticcircle.uconn.edu/SEEJ/ voisey/inuit.html>, (consulté le 20 février 2012).

LAPIERRE, D., et B. BRADSHAW, 2008 : Corporate Rationales for Negotiating Impact and Benefit Agreements. Communication au Canadian Institute of Mining, Metallurgy and Petroleum Annual Meeting, Technical Program, Edmonton. 
LOPPIE READING, C., et F. WIEN, 2009 : Health Inequities and Social Determinants of Aboriginal Peoples' Health. National Collaborating Centre for Aboriginal Health, Prince George.

MARMOT, M., 2001: «Economic and social determinants of disease ». Bulletin of the World Health Organization 79(10) : 988.

MARMOT, M., S. FRIEL, R. BELL, T.A. HOUWELING et S. TAYLOR, 2008: «Closing the gap in a generation: Health equity through action on the social determinants of health». The Lancet 372(9650): 1661-1669.

MONTENEGRO, R.A., et C. STEPHENS, 2006: «Aboriginal health in Latin America and the Caribbean». Lancet 367(9525) : 1859-1869.

NOBLE, B.F, et J.E. BRONSON, 2005: «Integrating human health in environmental impact assessment: Case studies of Canada's northern mining resource sector ». Arctic 58(4): 395-405.

NUNATSIAVUT GOVERNMENT, s.d. : Highlights of the Labrador Inuit Land Claims Agreement. Nunatsiavut Government, Nain, NFLD. Disponible sur Internet : <http://www.nunatsiavut.com/ images/stories/nunsgovernment/lilca/lilca_highlights.pdf>, (consulté le 20 février 2012).

O'FAIRCHEALLAIGH, Ciaran, 1999: " Making social impact assessment count: A negotiation-based approach for Indigenous Peoples ». Society \& Natural Resources 12(1) : 63-80.

—, 2006: «Mining agreements and Aboriginal economic development in Australia and Canada ». Journal of Aboriginal Economic Development 5(1) : 74-91.

-, s.d. : Reflections on the Sharing of Benefits from Australian Impact Benefit Agreements (IBAs). Disponible sur Internet: <http:// landkeepers.ca/images/uploads/reports/Reflections_on_sharing_of_benefits_from_Australian_IBAs_OFaircheallaigh.pdf>, (consulté le 21 février 2012).

ONSA (Organisation nationale de la santé autochtone), 2008 : An Overview of Traditional Knowledge and Medicine and Public Health in Canada. National Aboriginal Health Organization, Ottawa. Disponible sur Internet: <http://www.naho.ca/ documents/naho/publications/tkOverviewPublicHealth.pdfs, (consulté le 12 mars 2012).

PALMER, C.J., et al., 2002 : «HIV prevalence in a gold mining camp in the Amazon region, Guyana ». Emerging Infectious Diseases 8(3) : 330-331.

PRNO, Jason, 2007 : Assessing the Effectiveness of Impact and Benefit Agreements from the Perspective of their Aboriginal Signatories. Mémoire de maîtrise, département de géographie, Université de Guelph, Ontario.

PRNO, Jason, et Ben BRADSHAW, 2008 : «Program evaluation in a northern, Aboriginal setting: Assessing Impact and Benefit Agreements ». Journal of Aboriginal Economic Development 6(1): 59-75.

RICHMOND, Chantal A.M., 2009: " The social determinants of Inuit health: A focus on social support in the Canadian Arctic ». International Journal of Circumpolar Health 68(5) : 471-487.
RICHMOND, Chantal A.M., et Nancy A. ROSS, 2009: "The determinants of First Nation and Inuit health: A critical population health approach ». Health \& Place 15(2) : 403-411.

RNCAN (Ressources naturelles Canada), s.d. [a] : «Le projet Voisey's Bay: un exemple de réussite à Terre-Neuve-etLabrador quant à l'engagement des Autochtones dans un projet », dans L'engagement des Autochtones dans les secteurs des mines et de l'énergie. Ressources naturelles du Canada. Disponible sur Internet : <http://www.rncan.gc.ca/minerauxmetaux/autochtones/engagement/4217>, (consulté le 20 février 2012).

—, s.d. [b] : Ententes entre des sociétés minières et des collectivités autochtones ou des gouvernements. Disponible sur Internet: $<$ http://www.rncan.gc.ca/sites/www.nrcan.gc.ca.mineralsmetals/files/pdf/mms-smm/abor-auto/pdf/agr-ent-10-fra.pdf>, (consulté le 20 février 2012).

—, 2009 : Participation des Autochtones à l'industrie minière - Profil statistique. Bulletin d'information, Gouvernement du Canada, Ottawa. Disponible sur Internet: <http://www.rncan.gc.ca/ mineraux-metaux/autochtones/bulletins/3246>, (consulté le 17 février 2012).

SCALES, M., 2010 : «Canadian Mining Sector Partnerships with Aboriginals Create Enormous Benefits ». Canadian Mining Journal Daily News, March 29.

SIEBENMORGEN, Peter, 2009 : Developing an ideal mining agenda: Impact and Benefit Agreements as instruments of community development in Northern Ontario. Mémoire de maîtrise, département de géographie, Université de Guelph, Ontario.

SIEBENMORGEN, P., et B. BRADSHAW, 2011 : « Re-conceiving Impact and Benefit Agreements as instruments of Aboriginal community development in Northern Ontario, Canada ». Oil, Gas and Energy Law 4. Special issue on Indigenous People and Resources Development.

SHARMA, S., et S. REES, 2007 : «Consideration of the determinants of women's mental health in remote Australian mining towns ». The Australian Journal of Rural Health 15(1) : 1-7.

SOSA, I., et K. KEENAN, 2001 : Impact Benefit Agreements between Aboriginal Communities and Mining Companies: Their Use in Canada. Report, Canadian Environmental Law Association, Environmental Mining Council of British Columbia, CooperAcción. Disponible sur Internet : < http://s.cela.ca/files/ uploads/IBAeng.pdf>, (consulté le 20 février 2012).

STATISTIQUE CANADA, 2008 : Peuples autochtones du Canada en 2006: Inuits, Métis et Premières nations, Recensement de 2006 : résultats. $\mathrm{N}^{\circ}$ 97-558, ministre de l'Industrie, Ottawa.

STEEN, R., et al., 2000 : «Evidence of declining STD prevalence in a South African mining community following a core-group intervention ». Sexually Transmitted Diseases 27(1) : 1-8.

TELEWIAK, Robert, 2001 : «The Raglan Mine: What comes after an Impact and Benefit Agreement (IBA)?» Aboriginal Engagement and Sustainability: 63-72.

WILLIAMS, G. H., 2003 : « The determinants of health: Structure, context and agency ». Sociology of Health and Illness 25(3): 131-154. 